\title{
COMPORTAMENTO INGESTIVO DE NOVILHAS SUPLEMENTADAS A PASTO SOB NUTRIÇÃO COMPENSATÓRIA
}

\author{
INGESTIVE BEHAVIOR OF SUPPLEMENTED GRAZING HEIFERS ON COMPENSATORY \\ NUTRITION
}

\author{
Santana Júnior, H.A. ${ }^{1 *}$; Silva, R.R. ${ }^{2}$; Carvalho, G.G.P. ${ }^{3}$; Cardoso, E.O. ${ }^{2}$; Mendes, F.B.L. ${ }^{2}$; \\ Pinheiro, A.A. ${ }^{4}$; Abreu Filho, G. ${ }^{2}$; Dias, D.L.S. ${ }^{2}$; Barroso, D.S. ${ }^{2}$; Silva, F.F. ${ }^{2}$ \\ e Trindade Júnior, $\mathbf{G}^{2}$
}

\begin{abstract}
${ }^{1}$ UESPI. Campus Jesualdo Cavalcanti. Corrente-PI. Brasil. *hsantanajunior@hotmail.com ${ }^{2} \mathrm{DEBI} / \mathrm{UESB}$. Itapetinga-BA. Brasil.

3DPA/UFBA. Salvador-Bahia. Brasil. gleidsongiordano@yahoo.com.br 4PPZ/UEM. Maringá, Paraná. Brasil. jagualyson@hotmail.com
\end{abstract}

\section{PalaVRas ChaVE ADICIONAIS}

Bovino. Consumo. Etologia. Pastejo. Ruminante.

\author{
AdDitionAL KEYWORDS \\ Cattle. Ethology. Grazing. Intake. Ruminants.
}

\section{RESUMO}

O estudo foi realizado com o objetivo de avaliar os efeitos da nutrição compensatória em novilhas suplementadas a pasto sobre o comportamento ingestivo. O experimento foi conduzido na fazenda Princesa do Mateiro, município de Ribeirão do Largo, Bahia. Foram utilizadas 20 novilhas com grau de sangue 5/8 Guzerá Leiteiro 3/8 Holandês, com média de 18 meses de idade e peso corporal médio de $187 \pm 13,07 \mathrm{~kg}$. O experimento teve duração de 224 dias e os animais foram mantidos em sistema de produção a pasto, em pastejo rotacionado de Brachiaria brizantha 'Marandú'. Os animais foram aleatoriamente alocados em cada um dos tratamentos: Controle (T100): Animais recebendo concentrado para suprir $100 \%$ da exigência de nutrientes para ganho de $750 \mathrm{~g} / \mathrm{dia}$; Nutrição compensatória (NC): Animais que alternaram o nível de alimentação entre os períodos, sendo que nos períodos de restrição receberam concentrado para suprir $80 \%$ da exigência de nutrientes digestíveis totais do tratamento controle (T80) e nos períodos de realimentação receberam concentrado para suprir $120 \%$ das exigências de nutrientes digestíveis totais do tratamento controle (T120). Nos períodos de restrição, as médias do tempo de pastejo foram maiores para o NC (533 vs. 426 minutos) $(p<0,05)$. Os tempos de pastejo e ruminação, nos períodos de realimentação foram inferiores nos animais do grupo NC $(p<0,05)$. Durante a realimentação, o número de períodos de permanência no cocho e tempo por período de outras atividades foram maiores nos animais do tratamento $N C(p<0,05)$. No período de restrição, não houve diferenças estatísticas entre o controle e a nutrição compensatória para nenhuma das variáveis dos bocados e deglutição $(p>0,05)$. No período de realimentação, o número de bocados por deglutição tempo por deglutição, números de bocados por dia, os valores foram inferiores $(p<0,05)$ os animais do grupo $\mathrm{NC}$ em relação ao controle. $\mathrm{O}$ número de mastigações merícicas por dia e o número de bolos ruminados por dia foram diferentes entre o controle e a $N C(p<0,05)$. A nutrição compensatória altera o comportamento ingestivo de novilhas mestiças a pasto.

\section{SUMMARY}

The study was conducted to evaluate the effects of compensatory nutrition on supplemented pasture heifers on ingestive behavior. The experiment was conducted on the farm Princesa do Mateiro, Ribeirão do Largo, Bahia. Twenty 5/8 Guzera Dairy 3/8 Hostein heifers, with an average of 18 months of age and body weight of $187 \pm$ $13.07 \mathrm{~kg}$ were used. The experiment lasted 224 days and the animals were maintained in pasture 


\section{SANTANA JÚNIOR ETAL.}

production, rotational grazing Brachiaria brizantha 'Marandu'. The animals were randomly assigned to each of the treatments: control (T100): Animals fed concentrate to supply $100 \%$ of the requirement of nutrients to gain $750 \mathrm{~g} /$ day; compensatory nutrition (CN): animals that alternated the feed level periodically being restricted fed concentrate to meet the requirement of $80 \%$ total digestible nutrients of the control treatment (T80) and refeeding fed concentrate to supply $120 \%$ of the total digestible nutrient requirements of the control treatment (T120). In periods of restriction, the average grazing time was greater for NC (533 vs. 426 minutes) $(p<0.05)$. The grazing time and ruminating in feedback periods were lower than those of group NC $(p<0.05)$. During refeeding, the number of periods spent at the trough and period of time for other activities were higher in animals of treatment NC $(p<0.05)$. In the restricted period, there was no statistical differences between control and compensatory nutrition for the variables of bits and swallowing ( $p>0.05)$. During refeeding, the number of bits by swallowing, swallowing time and number of bites per day were lower $(p<0.05)$ in animals of NC group when compared to control. The number of chews per day and the number of ruminating bolus per day were different between the control and NC $(p<0.05)$. Compensatory nutrition alters the feeding behavior of crossbred heifers on pasture.

\section{INTRODUÇÃO}

A criação de novilhas para reposição do rebanho leiteiro é uma das etapas determinantes para o sucesso da atividade, devido seu elevado custo. Estimativas indicam que 15 a $20 \%$ do custo total da produção de leite são decorrentes de despesas com a criação de novilhas para reposição do rebanho e que aproximadamente $50 \%$ desse custo de produção resulta da alimentação dos animais (Costa et al., 2007). Um meio de reduzir os custos com a criação das novilhas é a aceleração do crescimento, propiciando a diminuição da idade ao primeiro parto e elevando a vida útil do animal.

Para a eficiente exploração da pastagem, é necessário conhecer as relações existentes na interface planta-animal, o que envolve o estudo de como as condições de pastejo interferem no comportamento ingestivo dos ruminantes e no seu desempenho, de forma a identificar condições de manejo adequadas à categoria animal e ao sistema de produção adotado (Jochims et al., 2010). O conhecimento do comportamento ingestivo dos animais de acordo com a dieta fornecida é de grande importância para avaliação de seu desempenho produtivo (Cavalcanti et al., 2008; Missio et al., 2010).

O comportamento alimentar de ruminantes em pastejo tem sido utilizado para nortear e embasar diversas discussões relacionadas ao consumo e, consequentemente, ao desempenho dos animais. Estudos de avaliação do comportamento ingestivo de animais em pastejo sobre nutrição compensatória são escassos, o que torna esta estratégia de alimentação duvidosa quanto aos efeitos provocados sobre o comportamento ingestivo, principalmente em termos de tempo de pastejo e ruminação.

Com isso, objetivou-se com este estudo avaliar o efeito da nutrição compensatória sobre o comportamento ingestivo de novilhas mestiças suplementadas a pasto.

\section{MATERIALE MÉTODOS}

O experimento foi conduzido na fazenda Princesa do Mateiro, município de Ribeirão do Largo, localizado na região sudoeste do estado da Bahia. Foram utilizadas 20 novilhas mestiças com grau de sangue $5 / 8$ Guzerá Leiteiro 3/8 Holandês, com média de 18 meses de idade e peso corporal médio inicial de $187 \pm 13,07 \mathrm{~kg}$. O experimento teve duração de 224 dias, tendo seu início em 25 de maio de 2008 e finalizando no dia 4 de janeiro de 2009, sendo composto por quatro períodos de 56 dias. Os animais foram mantidos em sistema de produção a pasto, em pastejo rotacionado de Brachiaria brizantha 'Marandú' em área de 10 ha, divididos em quatro piquetes de áreas equivalentes, sendo que os mesmos foram vedados 30 dias antes do início do experimento.

Os animais foram pesados e aleato- 
riamente alocados em cada um dos tratamentos: Controle (T100): Animais recebendo concentrado para suprir $100 \%$ da exigência de nutrientes para ganho de $750 \mathrm{~g} / \mathrm{dia}$; Nutrição compensatória (NC): alternou-se entre restrição (animais recebendo concentrado para suprir $80 \%$ da exigência de nutrientes digestíveis totais (NDT) do tratamento controle-T80) e realimentação (animais recebendo concentrado para suprir $120 \%$ das exigências de NDT do tratamento controle - T120), sendo que os mesmos foram constituídos pelos mesmos animais (10 animais). Desse modo, no $1^{\circ}$ e $3^{\circ}$ período, os animais receberam suplementação referente ao T80, e no $2^{\circ}$ e $4^{\circ}$ período, receberam suplementação referente ao T120. O concentrado foi formulado de acordo com o NRC (2001).

As amostras da forragem foram obtidas através do pastejo simulado observando os animais experimentais conforme Johnson (1978), identificando-se o tipo de material consumido e coletando-se uma amostra semelhante ao alimento ingerido.

A pastagem foi avaliada a cada 28 dias. Para estimar a disponibilidade de matéria seca (MS), foram tomadas 12 amostras por piquete, cortadas ao nível do solo com um quadrado de $0,25 \mathrm{~m}^{2}$, conforme metodologia descrita por McMeniman (1997) (tabela I). Foi adotado o método de lotação contínua com mesma carga animal. Para reduzir a influência da variação de biomassa entre piquetes, as novilhas permaneceram em cada piquete por sete dias e, após esse período, os tratamentos se alternavam nos piquetes. As estimativas de biomassa residual diária (BRD) de matéria seca foram realizadas nos dois piquetes que ficaram vedados (gaiolas de exclusão), conforme o método da dupla amostragem (Wilm et al., 1994). A biomassa de forragem foi de acordo com Gardner (1986).

As áreas cercadas nos piquetes funcionaram como gaiolas de exclusão. Foi calculada a taxa de acúmulo de MS diária (TAD) pela equação proposta por Campbell (1966). A taxa de lotação (TL) foi calculada consi-
Tabela I. Proporções de ingredientes do concentrado por período (\%), na base da matéria natural, e avaliação da pastagem. (Proportions of ingredients in the concentrate for a period $(\%)$, the base of natural matter, and evaluation of pasture).

\begin{tabular}{|c|c|c|c|c|}
\hline & \multicolumn{4}{|c|}{ Período } \\
\hline & $1 \stackrel{\circ}{0}$ & $2^{\circ}$ & $3^{\circ}$ & $4^{\circ}$ \\
\hline Milho grão moído & 28,20 & 66,47 & 70,52 & 72,05 \\
\hline Farelo de soja & 34,08 & 26,75 & 25,92 & 25,21 \\
\hline Farelo de trigo & 35,20 & 5,28 & 0,08 & - \\
\hline Calcário calcítico & 1,32 & - & 0,67 & 0,84 \\
\hline Fosfato bicálcico & - & - & 0,51 & - \\
\hline Ureia & - & - & - & 0,36 \\
\hline Sal mineral ${ }^{1}$ & 1,20 & 1,50 & 2,30 & 1,54 \\
\hline DTMS & 3474 & 2967 & 1910 & 3898 \\
\hline DMSV & 2758 & 1902 & 1266 & 2158 \\
\hline DMSpd & 2686 & 2207 & 1193 & 2898 \\
\hline DFDNpd & 1563 & 1335 & 725 & 1637 \\
\hline BRD & 83 & 88 & 60 & 122 \\
\hline TL & 0,92 & 1,12 & 1,28 & 1,38 \\
\hline TAD & 30 & 23 & 19 & 80 \\
\hline OF & 33 & 26 & 8 & 39 \\
\hline AF & 39 & 31 & 15 & 46 \\
\hline Folha (\%) & 40,3 & 24,2 & 24,3 & 43,3 \\
\hline Colmo (\%) & 39,1 & 39,9 & 42,0 & 36,3 \\
\hline Material morto (\%) & 20,6 & 35,9 & 33,7 & 20,4 \\
\hline Relação folha:colmo & 1,03 & 0,61 & 0,58 & 1,19 \\
\hline
\end{tabular}

${ }^{1}$ Composição: Cálcio 140 g; fósforo 65 g; sódio 148 $\mathrm{g}$; magnésio $5 \mathrm{~g}$; enxofre $12 \mathrm{~g}$; cobalto $107 \mathrm{mg}$; cobre $1550 \mathrm{mg}$; iodo $150 \mathrm{mg}$; manganês $1400 \mathrm{mg}$; níquel 30 mg; selênio 18 mg; zinco 4500 mg; 1120 mg; flúor (máximo) 650 mg.

DTMS: Disponibilidade total de matéria seca $(\mathrm{kg} /$ ha); DMSV: Disponibilidade de Matéria seca verde (kg/ha); DMSpd: Disponibilidade de Matéria seca potencialmente digestível (kg/ha); DFDNpd: Disponibilidade de Fibra em detergente neutro potencialmente digestível (kg/ha); BRD: Biomassa residual diária ( $\mathrm{kg} \mathrm{MS/ha/dia);} \mathrm{TL:} \mathrm{Taxa} \mathrm{de} \mathrm{lotação}$ (UA/ha); TAD: Taxa de acúmulo diário (kg MS/ha/ dia); OF: Oferta de forragem (kg MS/100 kg PC/ dia); AF: Altura da forragem (cm).

derando a unidade animal (UA) como sendo $450 \mathrm{~kg}$ de PV, utilizando-se a seguinte fórmula: 


\section{SANTANA JÚNIOR ETAL.}

$$
\mathrm{TL}=(\mathrm{UAt}) / a ́ r e a
$$

em que:

$\mathrm{TL}=$ taxa de lotação, em UA/ha;

$\mathrm{UAt}=$ unidade animal total;

Área= área experimental total, em ha.

A matéria seca e a fibra em detergente neutro potencialmente digestível (MSpd, FDNpd) da pastagem foram obtidas conforme descrito por Paulino et al. (2006).

Para estimar a produção fecal, utilizouse o óxido de cromo como indicador externo, fornecido durante 12 dias, com sete dias para adaptação e regulação do fluxo de excreção do marcador e cinco dias para coleta das fezes. As amostras de fezes foram analisadas por espectrofotometria de absorção atômica (EAA) para dosagem de cromo, conforme Williams et al. (1962). A determinação da produção fecal foi realizada conforme a equação proposta por Smith e Reid (1955). Para determinação da digestibilidade utilizou-se como indicador interno a fibra em detergente ácido indigestível (FDAi) (Casali et al., 2008). O consumo foi calculado através da produção fecal e da digestibilidade (tabela III).

As análises químico-bromatológicas seguiram as metodologias de Silva e Queiroz (2002) e Detmann e Valadares Filho (2010) (tabela II). Os carboidratos totais (CHOT) foram obtidos pela equação de (Sniffen $e t$ al., 1992), enquanto os carboidratos nãofibrosos (CNF), pela diferença entre $\mathrm{CHOT}$ e FDNcp. Os teores de nutrientes digestíveis totais (NDT) foram calculados segundo Weiss (1999).

A avaliação do comportamento foi realizada no $28^{\circ}$ dia de cada período, totalizando então quatro avaliações, sendo feitas observações a cada 10 minutos conforme metodologia de Silva et al. (2008), por um período de 24 horas, a fim de identificar o tempo destinado ao pastejo, ruminação, alimentando no cocho e outras atividades. As variáveis comportamentais estudadas foram: tempo de pastejo (PAS), tempo de ruminação (RUM), tempo alimentando no cocho (COC) e tempo em outras atividades (OUT).

O tempo de alimentação total (TAT) e de mastigação total (TMT) foi determinado pelas equações abaixo:

Tabela II . Composições química da forragem coletada por meio do pastejo simulado (PS) $e$ do suplemento (SUP) nos respectivos periodos experimentais. (Chemical compositions of forage collected through simulated grazing (PS) and the supplement (SUP) in the respective periods).

\begin{tabular}{|c|c|c|c|c|c|c|c|c|}
\hline & \multicolumn{2}{|c|}{$1^{\circ}$} & \multicolumn{2}{|c|}{$2^{\circ}$} & \multicolumn{2}{|c|}{$3^{\circ}$} & \multicolumn{2}{|c|}{$4 \stackrel{\circ}{-}$} \\
\hline & PS & Sup & PS & Sup & PS & Sup & PS & Sup \\
\hline MS (\%) & 27,45 & 91,23 & 25,34 & 90,56 & 40,05 & 90,45 & 24,87 & 90,76 \\
\hline $\mathrm{PB}(\% \mathrm{MS})$ & 12,23 & 22,56 & 11,09 & 20,26 & 8,78 & 19,49 & 15,13 & 20,20 \\
\hline $\mathrm{EE}(\% \mathrm{MS})$ & 2,65 & 2,89 & 2,76 & 2,67 & 2,35 & 3,45 & 2,69 & 3,65 \\
\hline СHOT (\% MS) & 82,56 & 75,27 & 83,76 & 76,80 & 87,87 & 75,65 & 84,04 & 74,83 \\
\hline CNF (\% MS) & 20,78 & 58,71 & 18,56 & 57,42 & 14,78 & 55,65 & 17,34 & 54,83 \\
\hline FDNcp (\% MS) & 72,08 & 15,31 & 75,73 & 17,48 & 78,39 & 16,08 & 67,75 & 16,88 \\
\hline FDA (\% MS) & 32,05 & 4,89 & 34,98 & 5,11 & 37,62 & 5,17 & 30,97 & 5,52 \\
\hline Cinza (\% MS) & 5,78 & 4,95 & 6,09 & 5,05 & 6,20 & 5,03 & 5,89 & 55,15 \\
\hline NDT (\% MS) & 69,07 & 77,19 & 68,35 & 81,63 & 58,79 & 80,52 & 67,89 & 81,23 \\
\hline
\end{tabular}

MS: Matéria seca; PB: Proteína bruta; EE: Extrato etéreo; $\mathrm{CHOT}$ : Carboidratos totais; $\mathrm{CNF}$ : Carboidratos não-fibrosos; FDNcp: Fibra em detergente neutro corrigido para cinzas e proteína; FDA: Fibra em detergente ácido; NDT: Nutrientes digestíveis totais.

Archivos de zootecnia vol. 62, núm. 237, p. 64. 
NOVILHAS SOBNUTRIÇÃO COMPENSATÓRIA:COMPORTAMENTO INGESTIVO

Tabela III. Consumo alimentar de novilhas mestiças durante o período de restrição e realimentação, com seus respectivos coeficientes de variação $(C V, \%)$. (Dietary intake of crossbred heifers during the restriction and refeeding, with respective coefficients of variation $(\mathrm{CV}, \%)$ ).

\begin{tabular}{|c|c|c|c|c|c|c|}
\hline & \multicolumn{3}{|c|}{ Restrição } & \multicolumn{3}{|c|}{ Realimentação } \\
\hline & Controle & $\mathrm{NC}$ & CV & Controle & $\mathrm{NC}$ & $\mathrm{CV}$ \\
\hline CMSS (kg) & 2,60 & 2,41 & 5,0 & 2,71 & 3,25 & 5,0 \\
\hline $\operatorname{CMSF}(\mathrm{kg})$ & 2,78 & 3,03 & 8,5 & 3,25 & 2,66 & 13,0 \\
\hline CMST $(\mathrm{kg})$ & 5,20 & 4,98 & 4,8 & 5,96 & 5,91 & 6,5 \\
\hline CFDN $(\mathrm{kg})$ & 2,69 & 2,78 & 7,1 & 2,81 & 2,49 & 10,1 \\
\hline $\operatorname{CCNF}(\mathrm{kg})$ & 1,56 & 1,33 & 1,6 & 1,94 & 2,17 & 2,5 \\
\hline CPB (kg) & 0,75 & 0,68 & 3,1 & 0,99 & 1,03 & 5,1 \\
\hline CNDT $(\mathrm{kg})$ & 3,37 & 3,11 & 5,7 & 3,79 & 3,88 & 6,3 \\
\hline
\end{tabular}

CMSS: Consumo de MS do suplemento; CMSF: Consumo de MS da forragem; CMST: Consumo MS total; CFDN: Consumo de fibra em detergente neutro; CCNF: Consumo de carboidratos não fibrosos; CPB: Consumo de proteína bruta; CNDT: Consumo de nutrientes digestíveis totais.

$$
T A T=P A S+C O C
$$

em que:

PAS (minutos) $=$ tempo de pastejo

$\operatorname{COC}$ (minutos) $=$ tempo de alimentação no cocho.

$$
T M T=P A S+R U M+C O C
$$

em que:

PAS (minutos) = tempo de pastejo;

RUM (minutos)= tempo de ruminação;

$\operatorname{COC}$ (minutos) $=$ tempo de alimentação no cocho.

A discretização das séries temporais foi realizada diretamente nas planilhas de coleta de dados, com a contagem dos períodos discretos de pastejo, ruminação, outras atividades e alimentação no cocho, conforme descrito por Silva et al. (2006). Foram calculados as eficiências de alimentação da MS (Burger et al., 2000), FDN, NDT, CNF e $\mathrm{PB}$ e ruminação da MS (Burger et al., 2000) e FDN (Burger et al., 2000).

Observou-se o número de mastigações merícicas por bolo ruminado (MMB) e o tempo gasto para ruminação de cada bolo (TeB). As variáveis, número de bolo ruminado por dia (BOL), velocidade de mastigação (VeM), tempo por mastigação merícica (TMM) e mastigações merícicas por dia (MMnd) foram calculadas pelas equações abaixo:

$$
\mathrm{BOL}=\mathrm{RUM} / \mathrm{TeB}
$$

em que:

$\mathrm{BOL}=$ número por dia;

$R U M=$ tempo de ruminação em segundos por dia; $\mathrm{TeB}=$ tempo por bolo ruminado em segundos.

\section{$\mathrm{VeM}=\mathrm{MMB} / \mathrm{TeB}$}

em que:

VeM= mastigações merícicas;

$\mathrm{MMB}=$ número de mastigações merícicas por bolo; $\mathrm{TeB}=$ tempo por bolo em segundos.

\section{$\mathrm{TMM}=\mathrm{TeB} / \mathrm{MMB}$}

em que:

$\mathrm{TMM}=$ em segundos;

$\mathrm{TeB}=$ tempo por bolo em segundos;

$\mathrm{MMB}=$ número de mastigações merícicas por bolo.

$\mathrm{MMnd}=\mathrm{BOL}{ }^{*} \mathrm{MMB}$

em que:

MMnd= em número por dia;

$\mathrm{BOL}=$ número de bolos ruminados por dia;

$\mathrm{MMB}=$ número de mastigações merícicas por bolo.

Durante os mesmos períodos de avalia- 
ção do comportamento animal, quando os animais estavam em atividade de pastejo a mais de 30 minutos, foi registrada a taxa de bocado (TxB) dos animais de cada tratamento, estimada por meio do tempo gasto pelo animal para realizar 20 bocados (Hodgson, 1982). Para o cálculo da massa de bocado (MaB), dividiu-se o consumo diário de forragem pelo total de bocados diários (Jamieson e Hodgson, 1979).

O delineamento experimental utilizado foi inteiramente casualizado (DIC), com os tratamentos controle e nutrição compensatória (restrição e realimentação) e 10 repetições. Sendo assim, foram comparados as médias da restrição as do controle, e as médias da realimentação as do controle. Os resultados foram interpretados estatisticamente por meio de análise de variância e teste F a $5 \%$ de probabilidade, utilizando-se o SAEG. O modelo estatístico utilizado foi:

$$
Y_{i j k}=\mu+T_{i}+\varepsilon_{i j k}
$$

sendo:

$\mathrm{Y}_{\mathrm{ijk}}=0$ valor observado da variável;

$\mu=$ constante geral;

$\mathrm{T}_{\mathrm{i}}=$ efeito do tratamento $\mathrm{i}$;

$\varepsilon_{\mathrm{ijk}}=$ erro associado a cada observação.

\section{RESULTADOSEDISCUSSÃO}

Nos períodos de restrição, os tempos de pastejo (PAS) e alimentação total (TAT) foram maiores nos animais do grupo NC $(\mathrm{p}<0,05)$, no entanto as variáveis outras atividades (OUT), ruminação (RUM), alimentando no cocho (COC) e mastigação total (TMT), se assemelharam estatisticamente com o controle $(\mathrm{p}>0,05)$. O fornecimento de suplemento concentrado provoca variação no tempo destinado ao pastejo, sendo verificado que a diminuição da quantidade de suplemento concentrado fez com que os animais elevassem seu tempo de pastejo, e consequentemente o TAT, com o objetivo de suprir a ingestão de matéria seca diária e dos respectivos nutrientes para atender as exigências de mantença e ganho esperado. O tempo de PAS e RUM dos animais do tratamento com nutrição compensatória, nos períodos de realimentação foram inferiores $(\mathrm{p}<0,05)$ aos do grupo controle (tabela IV). Como já foi discutido anteriormente, o aumento do nível de concentrado provocou diminuição do PAS. A redução da variável RUM no tempo destinado a atividade $(20,8 \%)$, pode ter ocorrido devido à diminuição do consumo de FDN $(2,81 \mathrm{~kg}$ vs. $2,49 \mathrm{~kg})$, representando uma diferença de $11,39 \%$.

Os valores de tempo de pastejo para NC foram de 5,55 horas e 8,88 horas nos períodos de realimentação e restrição, respectivamente. Neste estudo o tempo de pastejo observado nos animais do grupo NC no período de restrição alimentar foi superior a oito horas quando a pastagem estava com média de oferta de forragem (20,5 kg de MS/ $100 \mathrm{~kg}$ PC/dia), disponibilidade de matéria seca verde de 2,01 t/ha, altura da forragem de $27 \mathrm{~cm}$ e relação folha: colmo de 0,81 . Com isso, o PAS dos animais submetidos a restrição foi suficiente para propiciar o elevação do consumo de forragem pelos animais em 8,25\% com relação aos animais do grupo controle (3,03 vs. 2,78).

Os valores de TAT e TMT verificados nos animais do grupo NC nos períodos de realimentação foram inferiores $(\mathrm{p}<0,05)$ aos do grupo controle. Esse efeito no TMT é consequência dessa variável ser calculada por meio dos tempos de pastejo, ruminação e alimentando no cocho, portanto a diminuição do PAS e RUM provocaram esse efeito. O tempo em outras atividades (OUT), na realimentação foi mais elevado $(\mathrm{p}<0,05)$ para a NC quando comprados com o controle (tabela IV).

O tempo alimentando no cocho (COC), tanto no período de restrição quanto no de realimentação, não apresentaram diferenças significativas $(\mathrm{p}>0,05)$. Foi verificado no presente estudo que os animais tendem a alterar a velocidade de consumo de concentrado em decorrência da quantidade que é fornecido diariamente, portanto quando um 
NOVILHASSOBNUTRIÇÃO COMPENSATÓRIA:COMPORTAMENTO INGESTIVO

Tabela IV. Comportamento ingestivo de novilhas mestiças a pasto suplementadas sob nutrição compensatória (NC). (Ingestive behavior of crossbred heifers on pasture supplemented in compensatory nutrition $(\mathrm{CN}))$.

\begin{tabular}{|c|c|c|c|c|c|c|c|c|}
\hline & \multicolumn{3}{|c|}{ Restrição } & \multicolumn{5}{|c|}{ Realimentação } \\
\hline & Controle & $\mathrm{NC}$ & $\mathrm{CV}$ & $P$ & Controle & NC & $C V$ & $P$ \\
\hline PAS (min) & $426^{b}$ & $533^{a}$ & 10,0 & 0,00000 & $430^{a}$ & $333^{b}$ & 13,2 & 0,00000 \\
\hline OUT (min) & 583 & 532 & 20,4 & 0,16488 & $582^{b}$ & $757^{a}$ & 19,3 & 0,00013 \\
\hline RUM (min) & 395 & 333 & 29,0 & 0,06897 & $389^{a}$ & $308^{b}$ & 30,8 & 0,02158 \\
\hline $\operatorname{coc}(\min )$ & 34 & 41 & 36,6 & 0,14565 & 38 & 41 & 29,3 & ns \\
\hline TAT (min) & $461^{b}$ & $574^{a}$ & 8,9 & 0,00000 & $468^{a}$ & $374^{b}$ & 12,5 & 0,00000 \\
\hline TMT (min) & 856 & 907 & 12,9 & 0,16488 & $857^{\mathrm{a}}$ & $682^{b}$ & 16,8 & 0,00013 \\
\hline NPP $\left(n^{\circ} \stackrel{)}{ }\right.$ & 8,0 & 7,1 & 31,9 & 0,27368 & 8,5 & 8,3 & 37,4 & ns \\
\hline NPO (nº) & $16,7^{\mathrm{a}}$ & $12,1^{b}$ & 30,8 & 0,00233 & 17,2 & 16,3 & 34,8 & ns \\
\hline NPR ( $\left.n^{\circ}\right)$ & 10,8 & 8,4 & 41,0 & 0,05729 & 11,0 & 10,6 & 40,2 & ns \\
\hline NPC (nํ) & 1,0 & 1,0 & 0,0 & - & $1,0^{\mathrm{b}}$ & $1,7^{a}$ & 51,3 & 0,00279 \\
\hline TPP (min) & 63,4 & 78,9 & 37,3 & 0,07238 & 54,9 & 47,9 & 37,3 & 0,25094 \\
\hline TPO (min) & 38,8 & 48,6 & 42,0 & 0,09964 & $37,6^{\mathrm{b}}$ & $58,3^{\mathrm{a}}$ & 59,7 & 0,02846 \\
\hline TPR (min) & 41,0 & 42,1 & 32,3 & ns & 38,2 & 32,5 & 35,6 & 0,15754 \\
\hline TPC (min) & 34,5 & 41,0 & 36,6 & 0,14565 & $38,0^{\mathrm{a}}$ & $28,2^{b}$ & 28,2 & 0,00193 \\
\hline TxB (n/seg.) & 0,70 & 0,57 & 32,8 & 0,05939 & 0,84 & 0,84 & 26,4 & ns \\
\hline MaB (g) & 0,16 & 0,17 & 22,5 & ns & 0,15 & 0,16 & 16,8 & ns \\
\hline $\mathrm{BDe}\left(\mathrm{n}^{\circ}\right)$ & 31,4 & 33,7 & 42,2 & ns & $39,5^{\mathrm{a}}$ & $26,8^{\mathrm{b}}$ & 37,5 & 0,00259 \\
\hline TDe (seg.) & 49,7 & 58,6 & 37,5 & 0,17386 & $50,7^{a}$ & $31,4^{b}$ & 38,5 & 0,00041 \\
\hline NBD $\left(n^{\circ}\right)$ & 17647 & 18195 & 30,4 & ns & $21107^{a}$ & $16755^{b}$ & 27,2 & 0,01107 \\
\hline
\end{tabular}

abMédias seguidas por letras minúsculas diferentes, na mesma linha, diferem estatisticamente a 0,05 de probabilidade pelo teste $\mathrm{F}$.

CV: Coeficiente de variação (\%); PAS: Tempo de pastejo; OUT: Tempo em outras atividades; RUM:Tempo de ruminação; COC: Tempo de alimentando no cocho; TAT: Tempo de alimentação total; TMT: Tempo de mastigação total; NPP: Número por período de pastejo; NPO: Número por período em outras atividades; NPR: Número por período de ruminação; NPC: Número por período de alimentando no cocho; TPP: Tempo por período de pastejo; TPO: Tempo por período em outras atividades; TPR: Tempo por período de ruminação; TPC: Tempo por período de alimentando no cocho; TxB: Taxa de bocados; MaB: Massa do bocado; BDe: Número de bocados por deglutição; TDe: Tempo por deglutição; NBD: Número de bocados por dia.

maior nível de suplemento concentrado é fornecido no cocho, pois os animais consomem mais rapidamente, como foi verificada uma velocidade de consumo do concentrado de $79 \mathrm{~g} \mathrm{MS} /$ minuto de alimentação no cocho. Por outro lado, quando uma menor proporção de suplemento concentrado ofertado, os animais consomem em uma menor velocidade (59 g MS/minuto), obtendo assim semelhantes médias para o tempo de alimentação no cocho.
No período de restrição, o número e tempo por período de pastejo, ruminação, alimentando no cocho e outras atividades não diferiram entre a nutrição compensatória e o controle ( $p>0,05)$, com exceção do NPO, que foi maior para o controle, apresentando valores de 16,7 e 12,1, respectivamente, para controle e NC (tabela IV). O aumento do consumo de concentrado proporciona um menor tempo de busca por forragem, e consequentemente menor tempo de rumi- 
nação, elevando assim o número de períodos em outras atividades.

Durante a realimentação o número de período alimentando no cocho (NPC) e tempo por período de outras atividades (TPO) foram maiores $(\mathrm{p}<0,05)$ nos animais do grupo NC. Este resultado foi devido à elevação no nível de suplementação, que ocasionou maior frequência ao cocho para o consumo total do concentrado, tendo em vista que os animais do grupo $\mathrm{NC}$ foram ofertados $20 \%$ a mais de NDT no concentrado. Para o TPC, o NC diferiu do controle na realimentação $(\mathrm{p}<0,05)$, apresentando uma diminuição de $25,79 \%$ (38,0 vs. 28,2). Os tempos por período são oriundos da divisão do tempo total dependido na atividade pelo número de períodos na mesma atividade, com isso essa diminuição do TPC verificada nos animais da NC foi oriunda do aumento no NPC. Como os animais do grupo NC, no período de realimentação, receberam maior nível de concentrado que o controle, esse efeito reflete no ajuste de consumo feito pelos animais, indicando que apenas um período de cocho não foi suficiente para o consumo total do concentrado, o que causou diminuição do tempo por período de cocho.

No período de restrição, não houve diferenças estatísticas entre o controle e a nutrição compensatória para as variáveis dos bocados e da deglutição $(p>0,05)$. No período de realimentação, o número de bocados por deglutição (BDe), tempo por deglutição (TDe) e números de bocados por dia (NBD) foram inferiores $(p<0,05)$ os animais do grupo NC em relação ao controle. O maior nível de concentrado oferecido aos animais da NC na realimentação provocou diminuição no consumo de pasto e no tempo de pastejo, fato que contribuiu para obtenção de menores números de bocados por dia, tendo em vista que não houve diferença na velocidade de consumo de pasto $(\mathrm{TxB})$.

Diferente dos valores encontrado no presente estudo, Bremm et al. (2008) avaliaram diferentes estratégias de suple- mentação (nível crescente, fixo e decrescente de suplementação ao longo do estudo) em novilhas de corte, e relataram que a massa de bocados diferiu entre as estratégias de suplementação e foi maior na estratégia com nível crescente de suplementação. Já Glienke et al. (2010), avaliando o comportamento ingestivo de bezerras de corte, encontraram maiores massa de bocados para maiores níveis de suplementação. No entanto, no presente estudo, não houve diferenças significativas entre $\mathrm{NC}$ e o controle entre os animais que saíram do período de restrição (menor nível de concentrado) para a realimentação (maior nível de concentrado).

No período de restrição, as mastigações merícicas por bolo (MMB) e o tempo por bolo (TBo) não apresentaram diferenças significativas $(p>0,05)($ tabela V). Entretanto Silva et al. (2010), avaliando a terminação de novilhos Nelore em pastagem de Brachiaria brizantha, encontraram diferenças na MMB para os diferentes níveis de suplementação.

Tanto no período de restrição como na realimentação, a variável velocidade de mastigação dos animais da NC apresentou média inferior $(\mathrm{p}<0,05)$ ao tratamento controle, enquanto o tempo de mastigação foi superior $(\mathrm{p}<0,05)$ ao controle. No período de restrição, o efeito na variável VeM ocorreu em virtude do maior consumo de forragem, o que acarretou maior teor de FDN da dieta, proporcionando uma mastigação mais lenta. Já o TeM ocorreu o inverso, tendo em vista, que a diminuição da velocidade de mastigação provoca aumento no tempo necessário para mastigação, que é o tempo necessário para rompimento da fibra efetiva do conteúdo ruminal.

O número de mastigações merícicas por dia (MMnd) e o números de bolos ruminados por dia (BOL) foram maiores no tratamento controle em relação ao NC ( $\mathrm{p}<0,05)$ em ambos os períodos. Sabendo-se que o tempo de ruminação foi semelhante entre o controle e o NC no período de restrição, a diferença 
Tabela $\boldsymbol{V}$. Aspectos da ruminação e as eficiências de alimentação e ruminação de novilhas mestiças a pasto suplementadas sob nutrição compensatória $(N C)$. (Aspects of rumination and efficiencies of feeding and rumination crossbred heifers on pasture supplemented in compensatory nutrition $(\mathrm{CN})$ ).

\begin{tabular}{|c|c|c|c|c|c|c|c|c|}
\hline & \multicolumn{3}{|c|}{ Restrição } & \multicolumn{5}{|c|}{ Realimentação } \\
\hline & Controle & NC & CV & $P$ & Controle & $\mathrm{NC}$ & CV & $P$ \\
\hline $\operatorname{VMB}\left(\mathrm{n}^{\circ}\right)$ & 50,2 & 50,1 & 10,5 & ns & 54,4 & 52,4 & 11,1 & 0,30953 \\
\hline TBo (s) & 46,0 & 48,1 & 9,5 & 0,13884 & 50,5 & 50,9 & 11,0 & ns \\
\hline $\operatorname{VeM}(\mathrm{n} \% / \mathrm{s})$ & $1,09^{a}$ & $1,04^{b}$ & 6,6 & 0,03266 & $1,08^{a}$ & $1,03^{b}$ & 5,3 & 0,01494 \\
\hline TeM (s) & $0,92^{b}$ & $0,96^{a}$ & 6,6 & 0,03660 & $0,93^{b}$ & $0,97^{a}$ & 5,0 & 0,01713 \\
\hline MMnd ( $n^{\circ}$ ) & $25489^{a}$ & $19330^{b}$ & 32,1 & 0,01017 & $25700^{a}$ & $20690^{\mathrm{b}}$ & 30,5 & 0,03089 \\
\hline $\mathrm{BOL}\left(\mathrm{n}^{\circ}\right)$ & $515^{a}$ & $384^{b}$ & 31,6 & 0,00575 & 473 & 408 & 34,2 & 0,18636 \\
\hline EAMS (g/min) & $11,0^{\mathrm{a}}$ & $9,1^{\mathrm{b}}$ & 8,3 & 0,00010 & $12,8^{\mathrm{b}}$ & $16,0^{\mathrm{a}}$ & 10,5 & 0,00019 \\
\hline EAFDN (g/min) & $6,2^{\mathrm{a}}$ & $4,7^{\mathrm{b}}$ & 9,9 & 0,00001 & $5,4^{\mathrm{b}}$ & $7,5^{\mathrm{a}}$ & 13,0 & 0,00004 \\
\hline EANDT (g/min) & $6,8^{\mathrm{a}}$ & $5,9^{b}$ & 8,9 & 0,00217 & $8,4^{b}$ & $10,3^{\mathrm{a}}$ & 11,1 & 0,00052 \\
\hline EACNF (g/min) & 2,9 & 2,7 & 6,6 & 0,05211 & $4,7^{b}$ & $5,3^{\mathrm{a}}$ & 9,0 & 0,00489 \\
\hline EAPB (g/min) & $1,5^{\mathrm{a}}$ & $1,3^{\mathrm{b}}$ & 7,7 & 0,00227 & $2,2^{b}$ & $2,7^{\mathrm{a}}$ & 9,5 & 0,00070 \\
\hline ERMS (g/min) & $12,8^{b}$ & $16,9^{\underline{a}}$ & 25,4 & 0,02504 & $16,8^{b}$ & $20,5^{\mathrm{a}}$ & 20,3 & 0,04400 \\
\hline ERFDN (g/min) & 7,1 & 8,5 & 28,2 & 0,16103 & $7,0^{\mathrm{b}}$ & $9,6^{\mathrm{a}}$ & 20,6 & 0,00273 \\
\hline
\end{tabular}

abMédias seguidas por letras minúsculas diferentes, na mesma linha, diferem estatisticamente a 0,05 de probabilidade pelo teste $\mathrm{F}$.

CV: Coeficiente de variação (\%); MMB: Número de mastigações merícicas por bolo; TBo: Tempo por bolo; VeM: Velocidade de mastigação; TeM: Tempo por mastigação; MMnd: Número de mastigações merícicas por dia; BOL: Número de bolos ruminados por dia; EAMS: Eficiências de alimentação de matéria seca; EAFDN: Eficiências de alimentação da fibra em detergente neutro; EANDT: Eficiências de alimentação dos nutrientes digestíveis totais; EACNF: Eficiências de alimentação dos carboidratos nãofibrosos; EAPB: Eficiências de alimentação da proteína bruta; ERMS: Eficiências de ruminação da matéria seca; ERFDN: Eficiências de ruminação da fibra em detergente neutro.

encontrada no MMnd foi decorrente da diferença somente no tempo por mastigação. Em contrapartida, a diferença para MMnd no período de realimentação foi oriundo das diferenças estatísticas do tempo de ruminação e por mastigação. As variáveis MMB e TBo foram semelhantes $(\mathrm{p}>0,05)$ entre os animais da $\mathrm{NC}$ e o do controle. A variável BOL apresentou médias similares entre o controle e a NC no período de realimentação $(p>0,05)$, sobretudo no período de restrição o tratamento controle foi superior ao NC $(\mathrm{p}<0,05)$. As eficiências de alimentação EAMS, EAFDN, EANDT e EAPB, no período de restrição, foram superiores nos animais do tratamento controle $(\mathrm{p}<0,05)$. O efeito é justificado pela maior densidade de nutrientes na dieta dos animais do grupo citado, em virtude do maior nível de suplemento. Entretanto para EACNF não foi verificado diferenças entre o controle e o NC ( $p>0,05)$. No período de realimentação, verificaram-se diferenças entre todas as eficiências de alimentação e ruminação $(p<0,05)$, sendo os valores superiores os da NC que nesse período apresentou maior densidade dos nutrientes na dieta. A média da ERMS, na restrição, foi superior $(p<0,05)$ para a nutrição compensatória. AERFDN, não apresentou diferenças $(p>0,05)$ entre os animais do grupo controle e NC.

Destaca-se que a pouca citação de referências na discussão é decorrente da 


\section{SANTANA JÚNIOR ETAL.}

escassez de pesquisas de comportamento ingestivo com nutrição compensatória, sendo assim, sugere-se mais pesquisas na área para melhor avaliação, consolidando os efeitos.

\section{CONCLUSÃO}

A nutrição compensatória altera o

\section{BIBLIOGRAFIA}

Bremm, C.; Rocha, M.G.; Freitas, F.K.; Macari, S.; Elejalde, D.A.G. e Roso, D. 2008. Comportamento ingestivo de novilhas de corte submetidas a estratégias de suplementação em pastagens de aveia e azevém. Rev Bras Zootecn, 37: 1161-1167.

Burger, P.J.; Pereira, J.C.; Queiroz, A.C.; Silva, J.F.C.; Valadares Filho, S.C.; Cecon, P.R. e Casali, A.D.P. 2000. Comportamento ingestivo de bezerros holandeses alimentados com dietas contendo diferentes níveis de concentrado. Rev Bras Zootecn, 29: 236-242.

Campbell, A.G. 1966. Grazed pastures parameters; I. Pasture dry-matter production and availability in a stocking rate and grazing management experiment with dairy cows. $J$ Agric Sci, 67: 211-216.

Cavalcanti, M.C.A.; Batista, A.M.V.; Guim, A. e Lira, M.A. 2008. Consumo e comportamento ingestivo de caprinos e ovinos alimentados com palma gigante (Opuntia ficus-indica Mill) e palma orelha-de-elefante (Opuntia sp.). Acta Sci Anim Sci, 30: 173-179.

Casali, A.O.; Detmann, E.; Valadares Filho, S.C.; Pereira, J.C.; Henriques, L.T.; Freitas, S.G. e Paulino, M.F. 2008. Influência do tempo de incubação e do tamanho de partículas sobre os teores de compostos indigestíveis em alimentos e fezes bovinas obtidos por procedimentos in situ. Rev Bras Zootecn, 37: 335-342.

Costa, P.B.; Queiroz, A.C.; Rodrigues, M.T.; Magalhães, L.R.; Costa, M.G.; Toral, F.L.B.; Carvalho, T.A.; Monteiro, L.; Zorzi, K. e Duarte, M.S. 2007. Desempenho de novilhas leiteiras sob manejo para crescimento compensatório recebendo suplementação com ionóforo. Rev Bras Zootecn, 36: 461-470. comportamento ingestivo de novilhas mestiças a pasto. Os efeitos entre o período de restrição e realimentação são diferentes.

\section{AGRADECIMENTOS}

Ao Banco do Nordeste do Brasil pelo financiamento desta pesquisa.

Detmann, E. and Valadares Filho, S.C. 2010. On the estimation of non-fibrous carbohydrates in feeds and dietas. Arq Bras Med Vet Zoo, 62: 980-984.

Gardner, A.L. 1986. Técnicas de pesquisa em pastagem e aplicabilidade de resultados em sistema de produção. Brasília: IICA/EMBRAPA CNPGL. 197-205 pp.

Glienke, C.L.; Rocha, M.G.; Roso, D.; Potter, L.; Costa, V.G. and Machado, J.M. 2010. Ingestive behavior and displacement patterns of beef heifers on Italian ryegrass pasture. Rev Bras Zootecn, 39: 247-254.

Hodgson, J. 1982. Ingestive behavior. In: LEAVER, J.D. (Ed.). Herbage intake handbook. British Grassland Society. Hurley. UK. 113 pp.

Jamieson, W.S. and Hodgson, J. 1979. The effect of variation in sward characteristics upon the ingestive behavior and herbage intake of calves and lambs under continuous stocking management. Grass Forage Sci, 34: 273-281.

Jochims, F.; Pires, C.C.; Griebler, L.; Bolzan, M.S.; Dias, F.D. e Galvani, D.B. 2010. Comportamento ingestivo e consumo de forragem por cordeiras em pastagem de milheto recebendo ou não suplemento. Rev Bras Zootecn, 39: 572-581.

Johnson, A.D. 1978. Sample preparation and chemical analysis of vegetation. In: Manetje, L.T. (Ed.). Measurement of grassland vegetation and animal production. Commonwealth Agricultural Bureaux. Aberustwyth. 96-102 pp. McMeninan, N.P. 1997. Methods of estimating intake of grazing animals. Reunião Anual da Sociedade Brasileira de Zootecnia. Simpósio sobre tópicos especiais em Zootecnia, 34. Sociedade Brasileira de Zootecnia. Anais... Juiz de Fora. pp. 131-168.

Missio, R.L.; Brondani, I.L.; Alves Filho, D.L.; 


\section{NOVILHASSOB NUTRIÇÃO COMPENSATÓRIA:COMPORTAMENTO INGESTIVO}

Silveira, M.F.; Freitas, L.S. e Restle, J. 2010. Comportamento ingestivo de tourinhos terminados em confinamento, alimentados com diferentes níveis de concentrado na dieta. Rev Bras Zootecn, 39: 1571-1578.

NRC. 2001. National Research Council. Nutrient requirements of dairy cattle. National Academic Press. 7 ed. Washington, D.C. 381 pp.

Paulino, M.F.; Detmann, E. e Valadares Filho, S.C. 2006. Suplementação animal em pasto: energética ou protéica?. Simpósio sobre manejo estratégico da pastagem, 3. SIMFOR. Anais... Viçosa, MG. 359-392 pp.

Silva, D.J. e Queiroz, A.C. 2002. Análise de alimentos (métodos químicos e biológicos). Universidade Federal de Viçosa. Viçosa. 235 pp.

Silva, R.R.; Silva, F.F.; Prado, I.N.; Carvalho, G.G.P.; Franco, I.L.; Almeida, V.S.; Cardoso, C.P. e Ribeiro, M.H.S. 2006. Comportamento ingestivo de bovinos. Aspectos metodológicos. Arch Zootec, 55: 293-296.

Silva, R.R.; Prado, I.N.; Carvalho, G.G.P.; Santana Júnior, H.A.; Silva, F.F. e Dias, D.L.S. 2008. Efeito da utilização de três intervalos de observações sobre a precisão dos resultados obtidos no estudo do comportamento ingestivo de vacas leiteiras em pastejo. Ciênc Anim Bras, 9: 319-326.
Silva, R.R.; Prado, I.N.; Silva, F.F.; Almeida, V.V.S.; Santana Júnior, H.A.; Queiroz, A.C.; Carvalho, G.G.P. e Barroso, D.S. 2010. Comportamento ingestivo diurno de novilhos Nelore recebendo níveis crescentes de suplementação em pastejo de capim-braquiária. Rev Bras Zootecn, 39: 2073-2080.

Smith, A.M. and Reid, J.T. 1955. Use of chromic oxide as an indicator of fecal output for the purpose of determining the intake of a pasture herbage by grazing cows. J Dairy Sci, 38: 515524.

Sniffen, C.J.; O'connor, J.D.; Van Soest, P.J.; Fox, D.G. and Russel, J.B. 1992. A net carbohydrate and protein system for evaluating cattle diets: IICarbohydrate and protein availability. J Dairy Sci, 70: 3562-3577.

Weiss, W.P. 1999. Energy prediction equations for ruminant feeds. In: Cornell Nutrition Conference For Feed Manufacturers, 61. Cornell University. Proceedings... Ithaca. 176-185 pp.

Willians, C.H.; David, D.J. and Lilma, O. 1962. The determination of cromic oxide in faeces samples by atomic absorption spectrophotometry. J. Agric. Sci., 59: 381-385.

Wilm, H.G.; Costello, D.F. and Klipple, G.E. 1994. Estimating forage yield by the double sampling method. J Am Soc Agron, 36: 194-203. 